\title{
CASE REPORT \\ Hip abscess due to Aerococcus urinae in a man with paraplegia: case report
}

\author{
LL Goetz ${ }^{1,2}$, DJ Powell ${ }^{2}$, TA Castillo ${ }^{1,2}$, L Adkins $^{1}$, AP Klausner ${ }^{3,4}$ and DR Gater ${ }^{1,2}$
}

Study design: Single case report.

Objective: Present a case of hip abscess culture positive for Aerococcus urinae in a man with paraplegia.

Background: Aerococcus species are uncommonly reported and may be misinterpreted as alpha streptococci or staphylococci. This organism can cause significant morbidity due to urinary tract infection with septicemia or endocarditis.

Methods: Single case report.

Results: The patient required surgical incision and debridement. Open joint inspection was performed, which was complicated by superior dislocation. The patient later required a Girdlestone procedure.

Conclusions: A. urinae was cultured from a hip abscess in a man with paraplegia. Bacteremia, with the bladder as the reservoir, likely led to this abscess. Aerococcus is pathogenic and should be considered when culture results reveal unusual staph or strep species. Spinal Cord (2013) 51, 929-930; doi:10.1038/sc.2013.88; published online 17 September 2013

Keywords: spinal cord injuries; abscess; urinary tract infections; $A$. urinae

\section{INTRODUCTION}

Aerococcus is a rare pathogen first identified in 1992. Aerococci have been described as 'emerging human pathogens', which may be misidentified as alpha streptococci such as Viridans or Enterococcus. ${ }^{1}$ Clinical microbiological laboratories may consider it a skin contaminant, and recommend no further workup, even if obtained from catheterized urine. However, Aerococcus species can cause clinical infections including urinary tract infections (UTI). A PubMed search using the terms 'Aerococcus' and 'spinal cord injuries (SCI)' revealed no reports of cases in a person with SCI. We present a case of $A$. urinae cultured from a thigh abscess in a man with paraplegia.

\section{CASE REPORT}

A 38-year-old man with T3 AIS A paraplegia presented with lightheadedness, anorexia, nausea, vomiting, diarrhea and chills for 2 weeks. He had recently been treated with nitrofurantoin for Escherichia coli UTI at another facility. Vital signs were temperature $39.3^{\circ} \mathrm{C}$, pulse 125 b.p.m., blood pressure 142/90, respirations 18 . No thigh swelling was noted. Urinalysis revealed many white blood cells, red blood cells and trace leukocyte esterase and bacteria. Urine culture grew less than 10 thousand of three Gram-positive organisms, with no further workup recommended. Final culture result was ' 10 to 25 thousand non-enteric Streptococcus species', no further workup recommended.

Contrasted computed tomography scan of the abdomen and pelvis revealed a heterogeneous fluid/soft tissue density collection in the left hip measuring $11.1 \times 9.8 \times 16.9 \mathrm{~cm}$ extending into the joint space (Figure 1). The patient underwent operative incision and debridement with antibiotic coverage.
Intraoperative fluid culture revealed many white blood cells and moderate Gram-positive cocci in pairs. Final culture was 'moderate growth of Gram-positive cocci' identified as A. urinae, 'possible contaminant'. Antibiotic susceptibility testing revealed resistance to clindamycin but sensitivity to all other agents. Blood and fungal cultures were negative.

Repeat computed tomography scan at 1 month revealed an enhancing fluid collection and locules of gas consistent with an intra-articular abscess of the left hip, not significantly changed but with interval dislocation of the left hip (Figure 2) necessitating the left hip Girdlestone procedure. He also developed anemia (hemoglobin $=6.4 \mathrm{mg} \mathrm{dl}^{-1}$ ) requiring transfusion.

Over the course of his prolonged hospitalization, the patient required several intravenous antibiotics, both perioperatively and for abscess treatment, including cefazolin $2 \mathrm{~g}$ every $8 \mathrm{~h}$, ceftriaxone $1 \mathrm{~g}$ daily, vancomycin $1.5 \mathrm{~g}$ every $12 \mathrm{~h}$ and piperacillin/tazobactam $3.375 \mathrm{~g}$ every $8 \mathrm{~h}$.

\section{DISCUSSION}

This is the first report, to our knowledge, of Aerococcus infection in an individual with SCI. A review of Aerococci and Aerococcal infections has been recently published. ${ }^{1}$ Of the seven species identified, the most clinically important are A. urinae and $A$. sanguinicola. Both were first described as causes of UTI and later bacteremia, sepsis, endocarditis and spondylodiscitis. ${ }^{1}$

Predisposing factors for $A$. urinae infection reported in the scientific literature include male sex, age greater than 65 years and preexisting urinary tract pathology. Zhang et al. ${ }^{2}$ described two cases of UTI due to Aerococcus, a 'rarely reported pathogen, possibly due to difficulties in identification', absent from the databases of most

${ }^{1}$ Spinal Cord Injury \& Disorders Service, Hunter Holmes McGuire Veterans Affairs Medical Center, Richmond, VA, USA; ${ }^{2}$ Department of Physical Medicine and Rehabilitation, Virginia Commonwealth University, Richmond, VA, USA; ${ }^{3}$ Division of Urology, Department of Surgery, Hunter Holmes McGuire Veterans Affairs Medical Center, Richmond, VA, USA and ${ }^{4}$ Division of Urology, Department of Surgery, Virginia Commonwealth University, Richmond, VA, USA

Correspondence: Dr LL Goetz, Spinal Cord Injury \& Disorders Service, Hunter Holmes McGuire Veterans Affairs Medical Center, Richmond, VA 23249, USA

E-mail: lance.goetz@va.gov

Received 25 April 2013; revised 27 June 2013; accepted 2 July 2013; published online 17 September 2013 


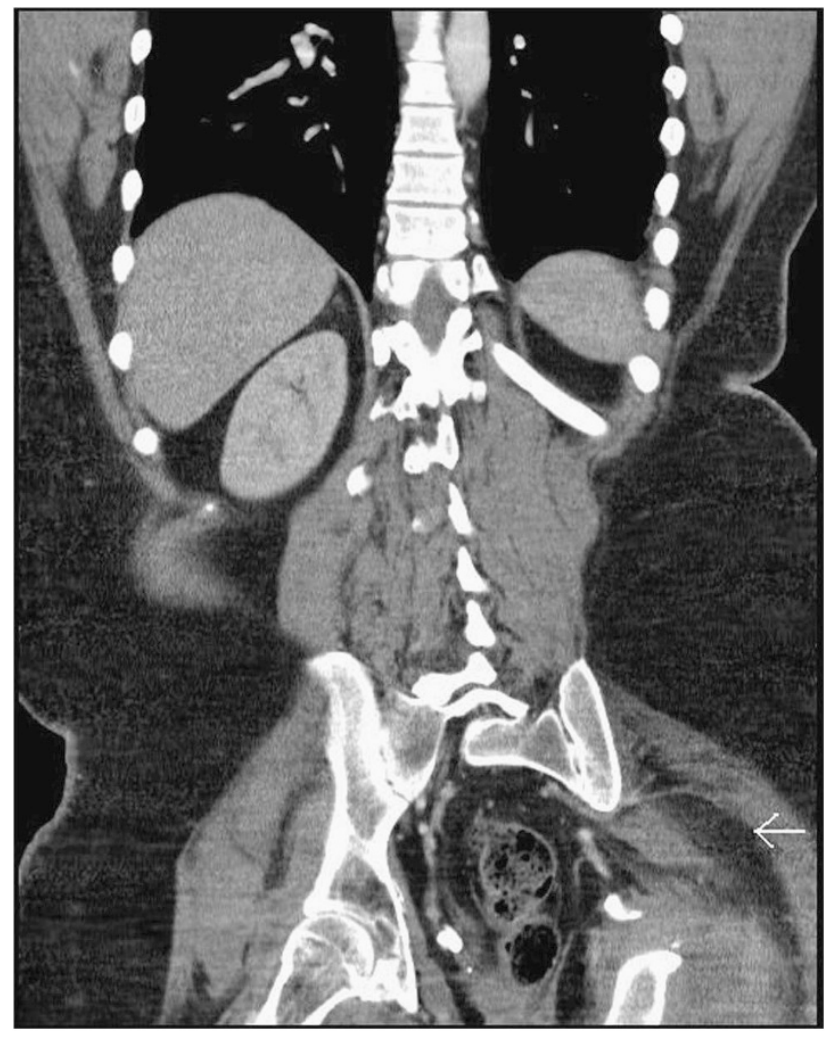

Figure 1 Frontal contrast-enhanced computed tomography images of the left hip demonstrating large heterogeneous fluid collection (arrow).

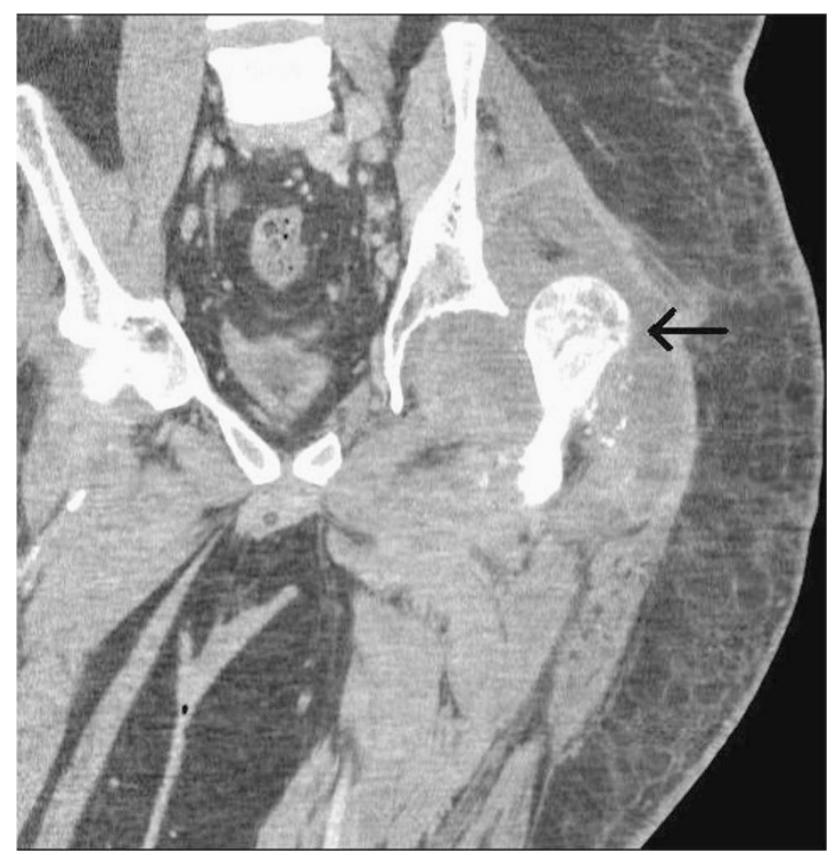

Figure 2 Frontal contrast-enhanced computed tomography image of the left hip demonstrating left hip dislocation. commercial systems and possibly misidentified as streptococcus, enterococcus or staphylococcus. Dysangco et al. ${ }^{3}$ reported two cases of endocarditis with valve vegetations due to A. urinae in young persons without urologic abnormality.

A. urinae is usually treated with a beta lactam and an aminoglycoside, with penicillins for less severe cases or penicillin or vancomycin with gentamicin for more severe cases. ${ }^{4}$ Sulfa resistance was often described for A. urinae, but recent literature reports most strains as susceptible to trimethoprim-sulfamethoxazole. ${ }^{5}$ Rasmussen ${ }^{1}$ suggests nitrofurantoin or fosfomycin for uncomplicated A. urinae UTI, and amoxicillin or a fluoroquinolone for complicated UTI.

Interestingly, another patient occupying the same room had a urine culture which grew Aerococcus species, not further identified, sensitive to all antibiotics. Repeat urine culture 2 months later grew 'alpha streptococcus not Group D or enterococcus' and 'less than 10000 Gram-positive organism, no further workup'. It is not known how the patient acquired the organism. His roommate presumably acquired the organism via cross-contamination from the patient. In addition to universal precautions including gowns and gloves, our facility cohorts for certain known resistant infectious agents, for example, methicillin-resistant $S$. aureus and Vancomycin-resistant Enterococci.

\section{CONCLUSION}

We conclude that A. urinae cultured from the patient's hip abscess was unlikely to be a contaminant, given the simultaneous urine culture with reported streptococcal species and A. urinae urine culture from another occupant of this patient's room. In light of the patient's recent UTI and urinary catheterization for bladder management, and no other known infectious sources, seeding of the hip from a bladder source is most likely. Our patient had a prolonged hospital course with significant morbidity, including two surgeries, hip dislocation and multiple antibiotics.

A urinae and Aerococcus species are pathogens, but may be misidentified or unreported. Appropriate identification and treatment is important to prevent significant morbidity or mortality. To our knowledge, this is the first report of Aerococcus infection in a person with SCI. Aerococcus is clearly pathogenic and should be considered when culture results reveal unusual staph or strep species. It remains to be seen whether Aerococcus will be cultured with increasing frequency in persons with SCI in the future.

\section{CONFLICT OF INTEREST}

The authors declare no conflict of interest.

1 Rasmussen M. Aerococci and aerococcal infections. J Infection 2013; 66: 467-474.

2 Zhang Q, Kwoh C, Attorri S, Clarridge JE. Aerococcus urinae in urinary tract infections. J Clin Microbiol 2000; 38: 1703-1705.

3 Dysangco A, Coronel RF, Li-Yu J, Purino FM, Sunarso S. Aerococcus urinae endocarditis: a report of two cases and review of literature. Philippine J Int Med 2010; 48: 48-52.

4 deVries TW, Brandenburg AH. Foul smelling urine in a 7-year-old boy caused by Aerococcus urinae. Ped Inf dis J 2012; 31: 1316-1317.

5 Shelton-Dodge K, Vetter EA, Kohner P, Nyre LM, Patel R.. Clinical significance and antimicrobial susceptibilities of Aerococcus sanguinicola and Aerococcus urinae. Diag Mic Inf Dis 2011; 70: 448-451. 